\title{
ERRATUM
}

\section{Erratum to: Effect of slow-release $\beta$-alanine tablets on absorption kinetics and paresthesia}

\author{
Jacques Décombaz • Maurice Beaumont • \\ Jacques Vuichoud · Florilene Bouisset • \\ Trent Stellingwerff
}

Published online: 17 July 2013

(C) Springer-Verlag Wien 2013

Erratum to: Amino Acids (2012) 43:67-76

DOI 10.1007/s00726-011-1169-7

In the original publication of Table 1, the last two entries of the first column have been published with incorrect units. The corrected Table 1 is produced below:

Table $1 \beta$-Alanine pharmacokinetic analysis

\begin{tabular}{|c|c|c|c|}
\hline & $\mathrm{TAB}^{\mathrm{a}}$ & $\mathrm{REF}^{\mathrm{b}}$ & $P$ \\
\hline$C_{\max }\left(\mu \mathrm{mol} \mathrm{L}{ }^{-1}\right)$ & $81.9 \pm 27.5$ & $248.2 \pm 112.7$ & 0.0002 \\
\hline$T_{\max }(\min )$ & $60.0 \pm 16.2$ & $29.4 \pm 7.2$ & 0.0015 \\
\hline $\operatorname{AUC}\left(\mu \mathrm{mol} \mathrm{L}{ }^{-1} \mathrm{~h}\right)$ & $229.4 \pm 83.7$ & $253.4 \pm 81.1$ & 0.5504 \\
\hline$K_{a}\left(\mathrm{~h}^{-1}\right)$ & $3.55 \pm 2.69$ & $11.98 \pm 7.21$ & 0.0007 \\
\hline$T_{\text {lag }}(\min )$ & $17.4 \pm 9.6$ & $14.4 \pm 4.2$ & 0.6606 \\
\hline$T_{1 / 2}(\min )$ & $80.4 \pm 64.2$ & $37.2 \pm 21.6$ & 0.0485 \\
\hline
\end{tabular}

Mean \pm SD

$C_{\max }$ peak concentration, $T_{\max }$ time to peak, $A U C$ area under the curve, $K_{a}$ absorption rate constant, $T_{\text {lag }}$ time until first appearance in plasma, $T_{1 / 2}$ half-time of disappearance

a $\beta$ A slow-release tablets

b $\beta$ A reference aqueous solution

The online version of the original article can be found under doi:10.1007/s00726-011-1169-7.

J. Décombaz $(\bowtie) \cdot$ M. Beaumont · J. Vuichoud · F. Bouisset ·

T. Stellingwerff

Nestlé Research Center, Lausanne, Switzerland

e-mail: decombaz.jac@bluewin.ch

Present Address:

T. Stellingwerff

Canadian Sports Centre-Pacific, Victoria, Canada 